\title{
BI-LEVEL OPTIMIZATION APPROACH FOR ROBUST MEAN-VARIANCE PROBLEMS
}

\author{
Pulak Swain*(D) And Akshay Kumar OJHa
}

\begin{abstract}
Portfolio Optimization is based on the efficient allocation of several assets, which can get heavily affected by the uncertainty in input parameters. So we must look for such solutions which can give us steady results in uncertain conditions too. Recently, the uncertainty based optimization problems are being dealt with robust optimization approach. With this development, the interest of researchers has been shifted toward the robust portfolio optimization. In this paper, we study the robust counterparts of the uncertain mean-variance problems under box and ellipsoidal uncertainties. We convert those uncertain problems into bi-level optimization models and then derive their robust counterparts. We also solve a problem using this methodology and compared the optimal results of box and ellipsoidal uncertainty models with the nominal model.
\end{abstract}

Mathematics Subject Classification. 90C17, 91-08, 91G10, 91G15.

Received November 24, 2020. Accepted August 14, 2021.

\section{INTRODUCTION}

Portfolio Optimization deals with the decision making problems of efficient distribution of financial assets. Markowitz mean-variance model $[17,18]$ is the base for this study which makes investment decisions in terms of mean and variance of returns. Some other risk models like semivariance, semiabsolute deviation, below target risk, value at risk, conditional value at risk have also come into picture when the return distribution is asymmetric $[9,10]$. In portfolio models we generally estimate the expected returns of individual assets from the past historical data. However, practically it is very difficult to forecast the accurate return due to several factors. If we ignore these uncertainties in our portfolio models, then our result could be very much different from the actual result. As a result, we may end up investing in an inefficient portfolio. So the uncertainty factor needs to be addressed properly and we should focus on making the solutions free from uncertainty. In the field of Optimization, such uncertainty based problems have been dealt with several approaches such as Stochastic programming, Sensitivity analysis, Dynamic programming, Fuzzy Optimization. However, these approaches cannot guarantee a completely uncertain free solution. In last two decades this problem has been addressed properly with the help of Robust optimization. It is first introduced by Ben-Tal for solving uncertain linear problems [2] and after that it is being used in many disciplines of science and engineering [5].

Keywords. Mean-variance model, box uncertainty, ellipsoidal uncertainty, robust optimization, bi-level optimization.

School of Basic Sciences, Indian Institute of Technology Bhubaneswar, Bhubaneswar 752050, Odisha, India.

*Corresponding author: ps28@iitbbs.ac.in 
In robust optimization, the uncertainty is based on a specific uncertain set. It assumes that the value of uncertain parameter perturbs around a nominal value and the set of all realizations of the uncertain parameter form a geometric structure like box, polyhedron, ellipsoid, paraboloid etc. [4]. The optimal solution which is feasible for any realization of the data uncertainty in the given set is computed through the Robust optimization approach. El Ghaoui and Lebret first studied the robust solutions to the uncertain least-squares problems [7], and El Ghaoui et al. studied uncertain semidefinite problems [8]. Later on Ben-Tal and Nemirovski [3] showed that when the uncertainty sets for a linear constraint are ellipsoids, the robust formulation turns out to be a conic quadratic problem. One of the first robust approaches to portfolio problems was made by Goldfarb and Iyengar [13], where they formulate robust portfolio problems as second order cone programs. Tütüncü and Koenig [21] present robust formulations when the expected return vector and the covariance matrix of asset returns are defined by lower and upper bounds and also illustrate how to compute the robust efficient frontier. Afterward Robust approach has been used very often in portfolio problems. Zhu et al. [22] studied the robust portfolio problems under a downside risk measure based on lower partial moment. The worst-case lower partial moments of degrees 0,1 , and 2 were formulated in terms of linear programs, second order conic programs and semidefinite programs. Fabozzi et al. [11] did a survey on robust mean-variance models as well as some robust downside risk models. They used value at risk and conditional value at risk for their study on downside risk. Lu [15] introduced a joint ellipsoidal uncertainty set in robust portfolio optimization. The study showed that the robust maximum risk-adjusted return problem with this uncertainty set can be solved as a conic programming problem. Dai and Wen [6] proposed a robust optimization method which minimizes the conditional value at risk of a portfolio under an affine data perturbation set. Fliege and Werner [12] studied the robust multiobjective optimization and they applied it in portfolio optimization by using a mean-variance problem. Kim et al. [14] analyzed the performance of robust portfolio for the US equity portfolios during the period of 1980 and 2014 . Their research confirms the effectiveness of robust optimization for controlling uncertainty in efficient investments. Asadujjaman and Zaman [1] studied the robust portfolio optimization under epistemic uncertainty using moment bounding approach and likelihood-based approach.

The robust problems are not so easy to solve, as here we have to first choose the worst case realization of uncertain parameters and then we solve the optimization problem with that worst case parameters. In the existing literature there is no proper methodology available for solving the robust counterparts of uncertain portfolio problems. Also a comparison among different uncertainty sets needs to be studied in terms of conservatism and optimality of their robust solutions. So in this paper our main focus is to propose a methodology for solving robust optimization problems and to compare the box and ellipsoidal uncertainty models. Our proposed methodology includes the transformation of uncertain mean-variance problems into bilevel optimization form and the use of single level reduction approach for solving this. Bilevel Optimization [19,20] consists of two-layered optimization problems where the upper level decision maker has complete knowledge of the lower level problem, while the lower level decision maker only optimizes its own problem. In this paper, we consider the uncertain parameters as the lower level decision variables to express the uncertain portfolio problems as bilevel optimization form. So in future using this approach many more research can be done in the field of robust optimization.

The organization of this paper is given as: Following the introduction part, a basics of mean-variance portfolio and its robustness have been discussed in Section 2. Section 3 presents the bilevel robust mean-variance problems under several uncertainties. We give some numerical illustrations in Section 4. Finally some concluding remarks have been incorporated in Section 5 .

\section{Preliminaries}

\subsection{Mean-variance portfolio model}

Consider an asset $i$ with the return over a period of time $t$ be $r_{i t}(t=1,2, \ldots, T)$. The Markowitz portfolio model is based on taking expected portfolio return as the reward and the variance of portfolio return as the risk factor. For that, first we need to calculate the expectation of returns of each individual asset and the 
covariance of returns between each pair of assets. Let $\mu_{i}$ be the expected value of return of $i$ th asset and $\sigma_{i j}$ be the covariance of return between $i$ th and $j$ th asset.

Mathematically we can write,

$$
\begin{aligned}
& \mu_{i}=E\left(r_{i}\right)=\frac{1}{T} \sum_{t=1}^{T} r_{i t} \\
& \sigma_{i j}=E\left[\left(r_{i}-\mu_{i}\right)\left(r_{j}-\mu_{j}\right)\right]=\frac{1}{T} \sum_{t=1}^{T}\left(r_{i t}-\mu_{i}\right)\left(r_{j t}-\mu_{j}\right) .
\end{aligned}
$$

The aim is to form a portfolio which will give our desired return with a minimum risk associated with it. Let the weight given to $i$ th asset be $x_{i}$. Then the expected return and variance of return of the portfolio are respectively given by,

$$
\mu_{P}=\sum_{i} \mu_{i} x_{i}, \quad \sigma_{P}^{2}=\sum_{i, j} \sigma_{i j} x_{i} x_{j}
$$

Markowitz Mean-Variance Model minimizes the variance of portfolio return at a given level of expected portfolio return (say $\tau$ ). Mathematically it can be formulated as:

$$
\begin{array}{ll}
\min & \frac{1}{2} \sum_{i, j} \sigma_{i j} x_{i} x_{j} \\
\text { s.t. } & \sum_{i} \mu_{i} x_{i} \geq \tau, \quad \sum_{i} x_{i}=1, \quad x_{i} \geq 0 .
\end{array}
$$

\subsection{Uncertainty in optimization problems and the robust counterpart}

An optimization problem is said to be uncertain when the coefficients of decision variables in the problem are not certain. That means their values perturb around a nominal value. Mathematically the general form of an uncertain optimization problem can be written as:

$$
\begin{array}{ll}
\min _{x} & f(\boldsymbol{x}, \boldsymbol{u}) \\
\text { s.t. } & c(\boldsymbol{x}, \boldsymbol{u}) \leq 0, \quad \forall \boldsymbol{u} \in \mathscr{U}(\boldsymbol{x})=\{\boldsymbol{u}: g(\boldsymbol{x}, \boldsymbol{u}) \leq 0\} .
\end{array}
$$

where $\boldsymbol{x} \in \mathbb{R}^{n}$ are decision variables, $\boldsymbol{u} \in \mathbf{R}^{p}$ are the uncertain parameters and $\mathscr{U}(\boldsymbol{x})$ be the uncertainty set. Since the uncertain parameters perturb around the nominal values, so we can represent the uncertain set in terms of some geometrical structures such as box, polyhedral, ellipsoid, paraboloid etc. Those sets can be formed by taking the nominal values as the coordinates of center and the perturbations are represented by the space between the center and boundaries. Some of those uncertain sets are given as follows:

1. Box uncertainty: Let $\boldsymbol{u}^{0}$ be the vector of nominal values of the uncertain parameters. Then the box uncertainty set can be given as:

$$
\mathscr{U}_{\mathrm{box}}=\left\{\boldsymbol{u}:\left\|\boldsymbol{u}-\boldsymbol{u}^{0}\right\|_{\infty} \leq \delta^{(B)}\right\}
$$

where $\|.\|_{\infty}$ is the supremum norm and $\delta^{(B)}$ is the maximum perturbation of the uncertain parameters.

2. Ellipsoidal uncertainty: Similarly the ellipsoidal uncertainty set can be represented as:

$$
\mathscr{U}_{\text {ellipsoidal }}=\left\{\boldsymbol{u}:\left\|\boldsymbol{u}-\boldsymbol{u}^{0}\right\|_{2} \leq \delta^{(E)}\right\}
$$

where $\|.\|_{2}$ is the euclidean norm. 
It is quite difficult to solve uncertain optimization problems, specially when the uncertainty set contains infinite number of elements. The main concern is that if we solve the problem by taking the nominal values, then the solution might be infeasible for many realization of uncertain parameters. So we cannot just ignore the uncertainty in the problem. In the last two decades the uncertain problems have been dealt with Robust Optimization approach, for its ability to find the solutions which are completely "immunized against uncertainty". Basically in Robust Optimization approach we solve the problem for the worst case realization of the uncertain parameters, so that the solution will be feasible for any realization of the uncertain parameters. First without loss of generality the objective function in (2.2) can always be transformed to a certain objective as:

$$
\begin{array}{ll}
\min _{t, \boldsymbol{x}} & t \\
\text { s.t. } & f(\boldsymbol{x}, \boldsymbol{u}) \leq t .
\end{array}
$$

So by assuming a certain objective function in problem (2.2), we can rewrite the general form of an uncertain problem as:

$$
\begin{array}{ll}
\min _{x} & f(\boldsymbol{x}) \\
\text { s.t. } & c(\boldsymbol{x}, \boldsymbol{u}) \leq 0, \quad \forall u \in \mathscr{U}(\boldsymbol{x})=\{\boldsymbol{u}: g(\boldsymbol{x}, \boldsymbol{u}) \leq 0\} .
\end{array}
$$

Then the robust counterpart of this problem can be written as:

$$
\begin{array}{ll}
\min _{x} & f(\boldsymbol{x}) \\
\text { s.t. } & \max _{\boldsymbol{u}}\{c(\boldsymbol{x}, \boldsymbol{u}): \boldsymbol{u} \in \mathscr{U}(\boldsymbol{x})\} \leq 0 .
\end{array}
$$

Since it is a minimization problem, so the worst case scenario can be obtained by taking those parameters from the uncertainty set which will give maximum values for the constraints. Similarly for a maximization problem, the worst case realization will be the minimum value of the uncertain parameter.

\subsection{Bi-level optimization}

Bi-Level optimization [16] consists of two levels of problems, where one problem is nested within the other. The general formulation of a bi-level optimization problem can be given by,

$$
\text { Upper Level: } \min _{x_{u} \in X_{U}, x_{l} \in X_{L}}\left\{F\left(x_{u}, x_{l}\right): G_{k}\left(x_{u}, x_{l}\right) \leq 0, \quad k=1,2, \ldots, K\right\}
$$

where $x_{l}$ is obtained from

$$
\text { Lower Level: } \quad x_{l} \in \underset{x_{l} \in X_{L}}{\operatorname{argmin}}\left\{f\left(x_{u}, x_{l}\right): g_{j}\left(x_{u}, x_{l}\right) \leq 0, \quad j=1,2, \ldots, J\right\}
$$

where $x_{u}$ and $x_{l}$ are respectively the upper and lower level decision variables. The argmin of a function is defined as the point(s) in the domain at which the function value is minimized.

In a similar fashion we can represent the robust counterpart of (2.2) as a bi-level problem given by,

$$
\begin{array}{ll}
\text { Upper Level: } & \min _{\boldsymbol{x}}\{f(\boldsymbol{x}, \boldsymbol{u}): c(\boldsymbol{x}, \boldsymbol{u}) \leq 0\} \\
\text { s.t. } & \\
\text { Lower Level: } & \boldsymbol{u} \in \underset{\boldsymbol{u}}{\operatorname{argmax}}\{c(\boldsymbol{x}, \boldsymbol{u}): g(\boldsymbol{x}, \boldsymbol{u}) \leq 0\}
\end{array}
$$

where argmax of a function gives the point(s) in the domain at which function is maximized.

When the lower level problem is convex and sufficiently regular, we can replace the lower level problem with its KKT conditions [20]. So the bi-level problem will be reduced to a single level constrained optimization 
problem:

$$
\begin{aligned}
& \min _{x} \quad f(\boldsymbol{x}, \boldsymbol{u}) \\
& \text { s.t. } \quad c(\boldsymbol{x}, \boldsymbol{u}) \leq 0 \\
& \nabla_{\boldsymbol{u}} L(\boldsymbol{x}, \boldsymbol{u}, \boldsymbol{\lambda})=0 \quad \text { where } L(\boldsymbol{x}, \boldsymbol{u}, \boldsymbol{\lambda})=c(\boldsymbol{x}, \boldsymbol{u})+\boldsymbol{\lambda}^{\top} g(\boldsymbol{x}, \boldsymbol{u}) \\
& g(\boldsymbol{x}, \boldsymbol{u}) \leq 0 \\
& \boldsymbol{\lambda} \perp g(\boldsymbol{x}, \boldsymbol{u}) \quad \text { (Complementary slackness condition) } \\
& \boldsymbol{\lambda} \geq 0 .
\end{aligned}
$$

\section{ROBUST MEAN-VARIANCE PROBLEMS UNDER SEVERAL UNCERTAINTIES BY BI-LEVEL OPTIMIZATION APPROACH}

As in portfolio optimization, we generally use the past historical data for evaluating the future return rates so there is a high chance of the solution to be influenced by uncertainty. And a small perturbation in data can mislead the investor to invest in an inefficient portfolio. Finding the robust solutions to the uncertain problems is not always straightforward. Here we propose a methodology for finding the robust solutions of such problems. The algorithm of our proposed methodology is as follows:

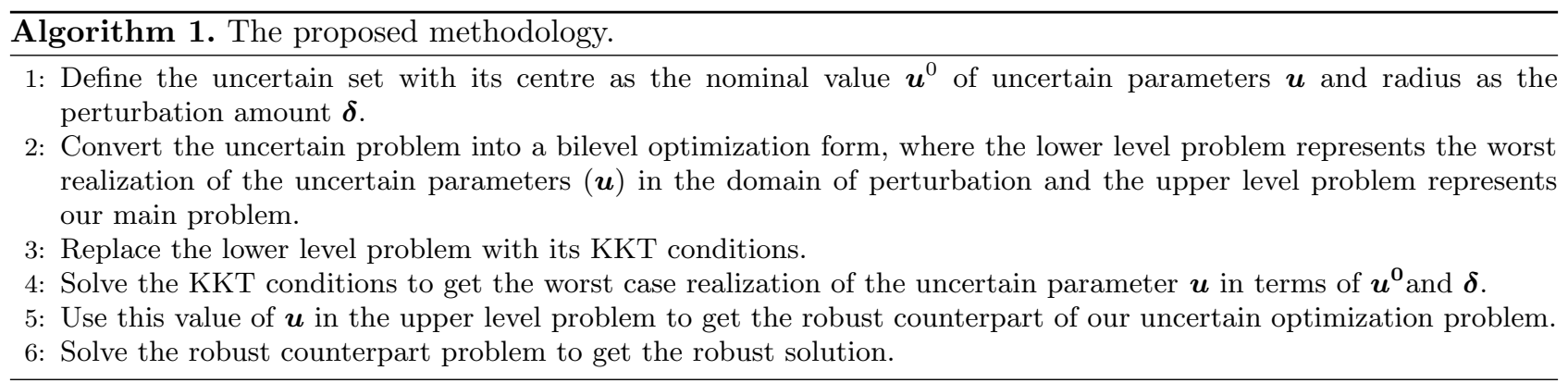

In mean-variance optimization, the perturbation may occur either in the mean return data or in the covariance matrix of the asset returns. In the following part we discuss how our methodology works to get the robust solutions of uncertain mean-variance optimization problems.

\subsection{When the expected returns of assets are uncertain}

Let us assume that the covariance terms are free from uncertainty and the uncertainty occurs only in expected return. Then the uncertain mean-variance portfolio problem is defined as:

$$
\begin{array}{ll}
\min _{x_{i}} & \frac{1}{2} \sum_{i, j} \sigma_{i j} x_{i} x_{j} \\
\text { s.t. } & \sum_{i} \mu_{i} x_{i} \geq \tau, \quad \sum_{i} x_{i}=1, \quad x_{i} \geq 0 \\
& \mu_{i} \in \mathscr{U}_{\boldsymbol{\mu}}
\end{array}
$$

where $\mathscr{U}_{\mu}$ is the uncertain set associated with the expected returns respectively. 


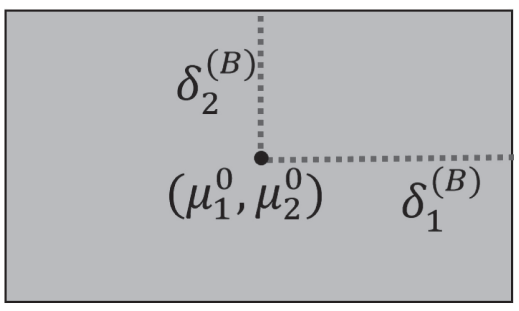

FiguRE 1. Box uncertainty set for a two-dimensional uncertain parameter $\boldsymbol{\mu}$.

The robust counterpart of the problem (3.1) is given by,

$$
\begin{array}{ll}
\min _{x_{i}} & \frac{1}{2} \sum_{i, j} \sigma_{i j} x_{i} x_{j} \\
\text { s.t. } & \left\{\min _{\mu_{i} \in \mathscr{U}_{\mu}} \sum_{i} \mu_{i} x_{i}\right\} \geq \tau, \quad \sum_{i} x_{i}=1 \quad x_{i} \geq \mathbf{0} .
\end{array}
$$

\subsubsection{Box uncertainty}

Theorem 3.1. Consider the problem (3.1). Let the expected returns $\mu_{i}(i=1,2, \ldots, n)$ are uncertain and they perturb within an $n$-dimensional box with center $\left(\mu_{1}^{0}, \mu_{2}^{0}, \ldots, \mu_{n}^{0}\right)$ and radius in ith dimension $\delta_{i}^{(B)}$.

Then the robust counterpart of the problem (3.1) is given by a quadratic programming problem,

$$
\begin{array}{ll}
\min _{x_{i}} & \frac{1}{2} \sum_{i, j} \sigma_{i j} x_{i} x_{j} \\
\text { s.t. } & \sum_{i}\left(\mu_{i}^{0}-\delta_{i}^{(B)}\right) x_{i} \geq \tau, \quad \sum_{i} x_{i}=1, \quad x_{i} \geq 0 .
\end{array}
$$

Proof. Assume that the uncertainty set associated with mean return is given by,

$$
\mathscr{U}_{\boldsymbol{\mu}}=\left\{\mu_{i}:\left|\mu_{i}-\mu_{i}^{0}\right| \leq \delta_{i}^{(B)}, \quad i=1,2, \ldots, n\right\}
$$

where $\mu_{i}^{0}$ is the nominal value of expected return for $i$ th asset and $\delta_{i}^{(B)}$ is the maximum perturbation that can happen.

The above inequality $\left|\mu_{i}-\mu_{i}^{0}\right| \leq \delta_{i}^{(B)}$ is equivalent to $\left(\mu_{i}-\mu_{i}^{0}\right)^{2} \leq\left(\delta_{i}^{(B)}\right)^{2}$, as both of these give the same set of solutions. So we can write the uncertainty set as:

$$
\mathscr{U}_{\boldsymbol{\mu}}=\left\{\mu_{i}:\left(\mu_{i}-\mu_{i}^{0}\right)^{2} \leq\left(\delta_{i}^{(B)}\right)^{2}, \quad i=1,2, \ldots, n\right\} .
$$

Then if we visualize the uncertain set $\mathscr{U}_{\boldsymbol{\mu}}$, it will look like a box with its center as the vector of nominal values $\left(\mu_{1}^{0}, \mu_{2}^{0}, \ldots, \mu_{n}^{0}\right)$ and its radius in $i$ th dimension be $\delta_{i}(B)$. And the expected return vector $\left(\mu_{1}, \mu_{2}, \ldots, \mu_{n}\right)$ can be any arbitrary point within the box.

It can be written in vector form as,

$$
\mathscr{U}_{\boldsymbol{\mu}}=\left\{\boldsymbol{\mu}:\left\|\boldsymbol{\mu}-\boldsymbol{\mu}^{0}\right\|_{\infty} \leq \boldsymbol{\delta}_{\boldsymbol{\mu}}^{(B)}\right\}
$$

where $\boldsymbol{\mu}^{0}=\left[\begin{array}{llll}\mu_{1}^{0} & \mu_{2}^{0} & \ldots & \mu_{n}^{0}\end{array}\right]^{\top}$ and $\boldsymbol{\delta}_{\boldsymbol{\mu}}^{(B)}=\left[\begin{array}{lll}\delta_{1}^{(B)} & \delta_{2}^{(B)} \ldots \delta_{n}^{(B)}\end{array}\right]^{\top}$ are respectively the vectors of nominal expected returns and their perturbations. 
Now we can transform equation (3.2) into bi-level form by taking the inner minimization problem as the lower level problem:

Upper Level: $\min _{x_{i}}\left\{\frac{1}{2} \sum_{i, j} \sigma_{i j} x_{i} x_{j}: \sum_{i} \mu_{i} x_{i} \geq \tau, \sum_{i} x_{i}=1, x_{i} \geq 0\right\}$

s.t.

Lower Level: $\quad \mu_{i} \in \underset{\mu_{i}}{\operatorname{argmin}}\left\{\sum_{i} \mu_{i} x_{i}:\left(\mu_{i}-\mu_{i}^{0}\right)^{2} \leq\left(\delta_{i}^{(B)}\right)^{2}\right\}$.

When the lower level problem is convex and sufficiently regular, we can replace the lower level problem with its KKT conditions [20].

Clearly the lower level problem in (3.3) is a convex problem, as its objective is linear and each component in the constraints is convex.

So replacing the lower level problem by its KKT conditions, we get

$$
\begin{aligned}
& \min _{x_{i}} \quad \frac{1}{2} \sum_{i, j} \sigma_{i j} x_{i} x_{j} \\
& \text { s.t.: } \quad \sum_{i} \mu_{i} x_{i} \geq \tau, \quad \sum_{i} x_{i}=1, \quad x_{i} \geq 0 \\
& \frac{\partial}{\partial \mu_{i}} L\left(x_{i}, \mu_{i}, \lambda_{i}\right)=0 \quad \text { where } L\left(x_{i}, \mu_{i}, \lambda_{i}\right)=\sum_{i} \mu_{i} x_{i}+\sum_{i} \lambda_{i}\left[\left(\mu_{i}-\mu_{i}^{0}\right)^{2}-\left(\delta_{i}^{(B)}\right)^{2}\right] \\
& \left(\mu_{i}-\mu_{i}^{0}\right)^{2} \leq\left(\delta_{i}^{(B)}\right)^{2}, \quad \lambda_{i}\left[\left(\mu_{i}-\mu_{i}^{0}\right)^{2}-\left(\delta_{i}^{(B)}\right)^{2}\right]=0, \quad \lambda_{i} \geq 0 \quad \text { for } i=1,2, \ldots, n .
\end{aligned}
$$

Now from the Lagrangian function $L$, we get

$$
\begin{gathered}
\frac{\partial L}{\partial \mu_{i}}=x_{i}+2 \lambda_{i}\left(\mu_{i}-\mu_{i}^{0}\right)=0 \\
\Longrightarrow \mu_{i}-\mu_{i}^{0}=\frac{-x_{i}}{2 \lambda_{i}} .
\end{gathered}
$$

So the single level form (3.4) can be given as:

$$
\begin{aligned}
& \min _{x_{i}} \quad \frac{1}{2} \sum_{i, j} \sigma_{i j} x_{i} x_{j} \\
& \text { s.t. } \quad \sum_{i} \mu_{i} x_{i} \geq \tau, \quad \sum_{i} x_{i}=1, \quad x_{i} \geq 0 \\
& \mu_{i}-\mu_{i}^{0}=\frac{-x_{i}}{2 \lambda_{i}} \\
& \left(\mu_{i}-\mu_{i}^{0}\right)^{2} \leq\left(\delta_{i}^{(B)}\right)^{2} \\
& \lambda_{i}\left[\left(\mu_{i}-\mu_{i}^{0}\right)^{2}-\left(\delta_{i}^{(B)}\right)^{2}\right]=0 \\
& \lambda_{i} \geq 0 .
\end{aligned}
$$

Here no $\lambda_{i}$ can be zero; otherwise from equation (3.5c) we will get, $\mu_{i}-\mu_{i}^{0} \rightarrow-\infty$.

So from equation (3.5e) we get, $\left(\mu_{i}-\mu_{i}^{0}\right)^{2}=\left(\delta_{i}^{(B)}\right)^{2}$. 


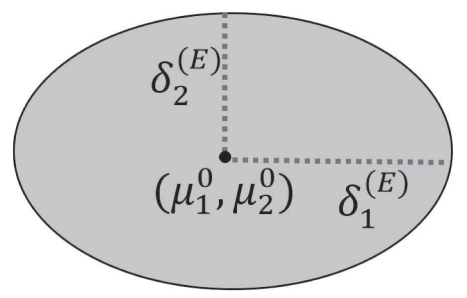

FIgURE 2. Ellipsoidal uncertainty set for a two-dimensional uncertain parameter $\boldsymbol{\mu}$.

Using equation (3.5c) we get, $\frac{x_{i}^{2}}{4 \lambda_{i}^{2}}=\left(\delta_{i}^{(B)}\right)^{2} \Longrightarrow \lambda_{i}=\frac{x_{i}}{2 \delta_{i}^{(B)}}$.

Then $\mu_{i}=\mu_{i}^{0}-\frac{x_{i}}{2 \lambda_{i}}=\mu_{i}^{0}-\delta_{i}^{(B)}$.

Hence our bilevel problem finally reduces to a quadratic programming problem:

$$
\begin{array}{ll}
\min _{x_{i}} & \frac{1}{2} \sum_{i, j} \sigma_{i j} x_{i} x_{j} \\
\text { s.t. } & \sum_{i}\left(\mu_{i}^{0}-\delta_{i}^{(B)}\right) x_{i} \geq \tau, \quad \sum_{i} x_{i}=1, \quad x_{i} \geq 0 .
\end{array}
$$

\subsubsection{Ellipsoidal uncertainty}

Theorem 3.2. Consider the problem (3.1). Let the expected returns $\mu_{i}(i=1,2, \ldots, n)$ are uncertain and they perturb within an $n$-dimensional ellipsoid with center $\left(\mu_{1}^{0}, \mu_{2}^{0}, \ldots, \mu_{n}^{0}\right)$ and radius in ith dimension be $\delta_{i}^{(E)}$. Then the robust counterpart of the problem (3.1) is given by a non-linear programming problem,

$$
\begin{array}{ll}
\min _{x_{i}} & \frac{1}{2} \sum_{i, j} \sigma_{i j} x_{i} x_{j} \\
\text { s.t. } & \sum_{i} \mu_{i}^{0} x_{i}-\sqrt{\sum_{i}\left(\delta_{i}^{(E)} x_{i}\right)^{2}} \geq \tau, \quad \sum_{i} x_{i}=1, \quad x_{i} \geq 0 .
\end{array}
$$

Proof. Let the uncertainty set associated with mean return is given by an ellipsoid like structure:

$$
\mathscr{U}_{\boldsymbol{\mu}}=\left\{\boldsymbol{\mu}:\left\|\boldsymbol{\mu}-\boldsymbol{\mu}^{0}\right\|_{2} \leq \boldsymbol{\delta}_{\boldsymbol{\mu}}^{(E)}\right\}
$$

Equivalently it can be represented as:

$$
\mathscr{U}_{\boldsymbol{\mu}}=\left\{\mu_{i}: \sum_{i=1}^{n} \frac{\left(\mu_{i}-\mu_{i}^{0}\right)^{2}}{\left(\delta_{i}^{(E)}\right)^{2}} \leq 1\right\}
$$


We can write equation (3.2) in bi-level form as:

$$
\text { Upper Level: } \min _{x_{i}}\left\{\frac{1}{2} \sum_{i, j} \sigma_{i j} x_{i} x_{j}: \sum_{i} \mu_{i} x_{i} \geq \tau, \sum_{i} x_{i}=1, x_{i} \geq 0\right\}
$$

s.t.

Lower Level: $\quad \mu_{i} \in \underset{\mu_{i}}{\operatorname{argmin}}\left\{\sum_{i} \mu_{i} x_{i}: \sum_{i=1}^{n} \frac{\left(\mu_{i}-\mu_{i}^{0}\right)^{2}}{\left(\delta_{i}^{(E)}\right)^{2}} \leq 1\right\}$.

Clearly the lower level problem in (3.7) has a convex objective function which is to be solved over the ellipsoidal set which is also convex itself. So the convexity property of lower level problem is satisfied and thus it can be replaced by its KKT conditions. That leads us to the following single objective problem:

$$
\begin{array}{ll}
\min _{x_{i}} & \frac{1}{2} \sum_{i, j} \sigma_{i j} x_{i} x_{j} \\
\text { s.t. } \quad \sum_{i} \mu_{i} x_{i} \geq \tau, \quad \sum_{i} x_{i}=1, \quad x_{i} \geq 0 & \\
\frac{\partial}{\partial \mu_{i}} L\left(x_{i}, \mu_{i}, \lambda_{i}\right)=0 \quad \text { where } L\left(x_{i}, \mu_{i}, \rho\right)=\sum_{i} \mu_{i} x_{i}+\rho\left[\sum_{i=1}^{n} \frac{\left(\mu_{i}-\mu_{i}^{0}\right)^{2}}{\left(\delta_{i}^{(E)}\right)^{2}}-1\right] \\
\sum_{i=1}^{n} \frac{\left(\mu_{i}-\mu_{i}^{0}\right)^{2}}{\left(\delta_{i}^{(E)}\right)^{2}} \leq 1, \quad \rho\left[\sum_{i=1}^{n} \frac{\left(\mu_{i}-\mu_{i}^{0}\right)^{2}}{\left(\delta_{i}^{(E)}\right)^{2}}-1\right]=0, \quad \rho \geq 0 .
\end{array}
$$

The Lagrangian function is given by,

$$
\begin{aligned}
L\left(x_{i}, \mu_{i}, \rho\right) & =\sum_{i} \mu_{i} x_{i}+\rho\left[\sum_{i=1}^{n} \frac{\left(\mu_{i}-\mu_{i}^{0}\right)^{2}}{\left(\delta_{i}^{(E)}\right)^{2}}-1\right] \\
\Longrightarrow \frac{\partial L}{\partial \mu_{i}} & =x_{i}+\frac{2 \rho\left(\mu_{i}-\mu_{i}^{0}\right)}{\left(\delta_{i}^{(E)}\right)^{2}}
\end{aligned}
$$

So equation (3.8) can be written as,

$$
\begin{aligned}
& \min _{x_{i}} \quad \frac{1}{2} \sum_{i, j} \sigma_{i j} x_{i} x_{j} \\
& \text { s.t. } \quad \sum_{i} \mu_{i} x_{i} \geq \tau, \quad \sum_{i} x_{i}=1, \quad x_{i} \geq 0 \\
& x_{i}+\frac{2 \rho\left(\mu_{i}-\mu_{i}^{0}\right)}{\left(\delta_{i}^{(E)}\right)^{2}}=0 \\
& \sum_{i=1}^{n} \frac{\left(\mu_{i}-\mu_{i}^{0}\right)^{2}}{\left(\delta_{i}^{(E)}\right)^{2}} \leq 1
\end{aligned}
$$




$$
\begin{aligned}
& \rho\left[\sum_{i=1}^{n} \frac{\left(\mu_{i}-\mu_{i}^{0}\right)^{2}}{\left(\delta_{i}^{(E)}\right)^{2}}-1\right]=0 \\
& \rho \geq 0 .
\end{aligned}
$$

If $\rho=0$, then from equation (3.9c) we get each $x_{i}=0$, which will contradict the normality condition $\sum_{i} x_{i}=1$.

Hence $\rho>0$. Then from (3.9e) we get, $\sum_{i=1}^{n} \frac{\left(\mu_{i}-\mu_{i}^{0}\right)^{2}}{\left(\delta_{i}^{(E)}\right)^{2}}=1$.

Now using equation (3.9c) we get, $\frac{1}{4 \rho^{2}} \sum_{i}\left(\delta_{i}^{(E)} x_{i}\right)^{2}=1 \Longrightarrow \rho=\frac{\sqrt{\sum_{i}\left(\delta_{i}^{(E)} x_{i}\right)^{2}}}{2}$.

Hence from equation (3.9c) we get, $\mu_{i}=\mu_{i}^{0}-\frac{\left(\delta_{i}^{(E)}\right)^{2} x_{i}}{2 \rho}=\mu_{i}^{0}-\frac{\left(\delta_{i}^{(E)}\right)^{2} x_{i}}{\sqrt{\sum_{i}\left(\delta_{i}^{(E)} x_{i}\right)^{2}}}$.

So the expected return of the portfolio becomes, $\sum_{i} \mu_{i} x_{i}=\sum_{i} \mu_{i}^{0} x_{i}-\sqrt{\sum_{i}\left(\delta_{i}^{(E)} x_{i}\right)^{2}}$.

Thus our bilevel problem finally reduces to the following second order conic programming problem:

$$
\begin{array}{ll}
\min _{x_{i}} & \frac{1}{2} \sum_{i, j} \sigma_{i j} x_{i} x_{j} \\
\text { s.t. } & \sum_{i} \mu_{i}^{0} x_{i}-\sqrt{\sum_{i}\left(\delta_{i}^{(E)} x_{i}\right)^{2}} \geq \tau, \quad \sum_{i} x_{i}=1, \quad x_{i} \geq 0 .
\end{array}
$$

\subsection{When the covariance terms are uncertain}

Now assume that the covariance matrix is uncertain and the expected return is free from uncertainty. Then the uncertain mean-variance portfolio problem is defined as:

$$
\begin{array}{ll}
\min _{x_{i}} & \frac{1}{2} \sum_{i, j} \sigma_{i j} x_{i} x_{j} \\
\text { s.t. } & \sum_{i} \mu_{i} x_{i} \geq \tau, \quad \sum_{i} x_{i}=1, \quad x_{i} \geq 0 \\
& \sigma_{i j} \in \mathscr{U}_{\boldsymbol{\sigma}} .
\end{array}
$$

where $\mathscr{U}_{\mu}$ is the uncertain set associated with the expected returns respectively.

The robust counterpart of the problem (3.11) is given by,

$$
\begin{array}{ll}
\min _{x_{i}} & \left\{\max _{\sigma_{i j} \in \mathscr{U}_{\sigma}} \frac{1}{2} \sum_{i, j} \sigma_{i j} x_{i} x_{j}\right\} \\
\text { s.t. } & \sum_{i} \mu_{i} x_{i} \geq \tau, \quad \sum_{i} x_{i}=1 \quad x_{i} \geq 0 .
\end{array}
$$

\subsubsection{Box uncertainty}

Theorem 3.3. Consider the problem (3.11). Let the covariance terms $\sigma_{i j}(i, j=1,2, \ldots, n)$ are uncertain and they perturb around the nominal values $\sigma_{i j}^{0}(i, j=1,2, \ldots, n)$ with a highest possible perturbation of 
$\Delta_{i j}^{(B)}(i, j=1,2, \ldots, n)$, then the robust counterpart of the problem (3.11) is given by a quadratic programming problem,

$$
\begin{array}{ll}
\min _{x_{i}} & \frac{1}{2} \sum_{i, j}\left[\sigma_{i j}^{0}+\Delta_{i j}^{(B)}\right] x_{i} x_{j} \\
\text { s.t. } & \sum_{i} \mu_{i} x_{i} \geq \tau, \quad \sum_{i} x_{i}=1, \quad x_{i} \geq 0 .
\end{array}
$$

where the uncertainty set of $\sigma_{i j}$ 's is a box of dimension $n^{2}$.

Proof. When the covariance terms of assets are associated with the box uncertainty set:

$$
\mathscr{U}_{\boldsymbol{\sigma}}=\left\{\sigma_{i j}:\left(\sigma_{i j}-\sigma_{i j}^{0}\right)^{2} \leq\left(\Delta_{i j}^{(B)}\right)^{2}, \quad i=1,2, \ldots, n, j=1,2, \ldots, n\right\} .
$$

We can write the problem (3.11) in bilevel form as:

Upper Level: $\min _{x_{i}}\left\{\frac{1}{2} \sum_{i, j} \sigma_{i j} x_{i} x_{j}: \sum_{i} \mu_{i} x_{i} \geq \tau, \sum_{i} x_{i}=1, x_{i} \geq 0\right\}$

s.t.

Lower Level: $\quad \sigma_{i j} \in \underset{\sigma_{i j}}{\operatorname{argmax}}\left\{\sum_{i, j} \sigma_{i j} x_{i} x_{j}:\left(\sigma_{i j}-\sigma_{i j}^{0}\right)^{2} \leq\left(\Delta_{i j}^{(B)}\right)^{2}\right\}$.

The lower level problem can be easily converted into a minimization problem:

Upper Level: $\min _{x_{i}}\left\{\frac{1}{2} \sum_{i, j} \sigma_{i j} x_{i} x_{j}: \sum_{i} \mu_{i} x_{i} \geq \tau, \sum_{i} x_{i}=1, x_{i} \geq 0\right\}$

s.t.

Lower Level: $\quad \sigma_{i j} \in \underset{\sigma_{i j}}{\operatorname{argmin}}\left\{-\sum_{i, j} \sigma_{i j} x_{i} x_{j}:\left(\sigma_{i j}-\sigma_{i j}^{0}\right)^{2} \leq\left(\Delta_{i j}^{(B)}\right)^{2}\right\}$.

Now here also we have a convex lower level problem. So we can transform the problem into the following single level problem,

$$
\begin{array}{ll}
\min _{x_{i}} & \frac{1}{2} \sum_{i, j} \sigma_{i j} x_{i} x_{j} \\
\text { s.t. } \quad & \sum_{i} \mu_{i} x_{i} \geq \tau, \quad \sum_{i} x_{i}=1, \quad x_{i} \geq 0 \\
\frac{\partial}{\partial \sigma_{i j}} L\left(x_{i}, x_{j}, \sigma_{i j}, \lambda_{i j}\right)=0 \quad \text { where } L\left(x_{i}, x_{j}, \sigma_{i j}, \lambda_{i j}\right)=-\sum_{i j} \sigma_{i j} x_{i} x_{j}+\sum_{i j} \lambda_{i j}\left[\left(\sigma_{i j}-\sigma_{i j}^{0}\right)^{2}-\left(\Delta_{i j}^{(B)}\right)^{2}\right] \\
\left(\sigma_{i j}-\sigma_{i j}^{0}\right)^{2} \leq\left(\Delta_{i j}^{(B)}\right)^{2}, \quad \lambda_{i j}\left[\left(\sigma_{i j}-\sigma_{i j}^{0}\right)^{2}-\left(\Delta_{i j}^{(B)}\right)^{2}\right]=0, \quad \lambda_{i j} \geq 0 \quad \text { for } i=1,2, \ldots, n, j=1,2, \ldots, n .
\end{array}
$$


Now from the Lagrangian function $L$, we get

$$
\begin{aligned}
& \frac{\partial L}{\partial \sigma_{i j}}=-x_{i} x_{j}+2 \lambda_{i j}\left(\sigma_{i j}-\sigma_{i j}^{0}\right)=0 \\
& \Longrightarrow \sigma_{i j}-\sigma_{i j}^{0}=\frac{x_{i} x_{j}}{2 \lambda_{i j}} .
\end{aligned}
$$

So the single level form (3.15) can be written as:

$$
\begin{aligned}
& \min _{x_{i}} \quad \frac{1}{2} \sum_{i, j} \sigma_{i j} x_{i} x_{j} \\
& \text { s.t. } \quad \sum_{i} \mu_{i} x_{i} \geq \tau, \quad \sum_{i} x_{i}=1, \quad x_{i} \geq 0 \\
& \sigma_{i j}-\sigma_{i j}^{0}=\frac{x_{i} x_{j}}{2 \lambda_{i j}} \\
& \left(\sigma_{i j}-\sigma_{i j}^{0}\right)^{2} \leq\left(\Delta_{i j}^{(B)}\right)^{2} \\
& \lambda_{i j}\left[\left(\sigma_{i j}-\sigma_{i j}^{0}\right)^{2}-\left(\Delta_{i j}^{(B)}\right)^{2}\right]=0 \\
& \lambda_{i j} \geq 0 .
\end{aligned}
$$

Here no $\lambda_{i j}$ can be zero; otherwise from equation (3.16c) we will get, $\sigma_{i j}-\sigma_{i j}^{0} \rightarrow-\infty$. So from equation (3.16e) we get, $\left(\sigma_{i j}-\sigma_{i j}^{0}\right)^{2}=\left(\Delta_{i j}^{(B)}\right)^{2}$.

Using equation (3.16c) we get, $\frac{\left(x_{i} x_{j}\right)^{2}}{4 \lambda_{i j}^{2}}=\left(\Delta_{i j}^{(B)}\right)^{2} \Longrightarrow \lambda_{i j}=\frac{x_{i} x_{j}}{2 \Delta_{i j}^{(B)}}$, as $\lambda_{i j}$ is non-negative so we take the positive square root.

Then from equation(3.16c) we get, $\sigma_{i j}=\sigma_{i j}^{0}+\Delta_{i j}^{(B)}$.

Hence our bilevel problem finally reduces to a quadratic programming problem:

$$
\begin{array}{ll}
\min _{x_{i}} & \frac{1}{2} \sum_{i, j}\left[\sigma_{i j}^{0}+\Delta_{i j}^{(B)}\right] x_{i} x_{j} \\
\text { s.t. } & \sum_{i} \mu_{i} x_{i} \geq \tau, \quad \sum_{i} x_{i}=1, \quad x_{i} \geq 0 .
\end{array}
$$

\subsubsection{Ellipsoidal uncertainty}

Theorem 3.4. Consider the problem (3.11). Let the covariance terms $\sigma_{i j}(i, j=1,2, \ldots, n)$ are uncertain and they perturb around the nominal values $\sigma_{i j}^{0}(i, j=1,2, \ldots, n)$ with a highest possible perturbation of $\Delta_{i j}^{(E)}(i, j=1,2, \ldots, n)$, then the robust counterpart of the problem (3.11) is given by the following non-linear programming,

$$
\begin{array}{ll}
\min _{x_{i}} & \frac{1}{2}\left[\sum_{i, j} \sigma_{i j}^{0} x_{i} x_{j}+\sqrt{\sum_{i, j}\left(\Delta_{i j}^{(E)} x_{i} x_{j}\right)^{2}}\right] \\
\text { s.t. } & \sum_{i} \mu_{i} x_{i} \geq \tau, \quad \sum_{i} x_{i}=1, \quad x_{i} \geq 0 .
\end{array}
$$

where the uncertainty set of $\sigma_{i j}$ 's is an ellipsoid of dimension $n^{2}$. 
Proof. When the covariance terms of assets are associated with the ellipsoidal uncertainty set:

$$
\mathscr{U}_{\boldsymbol{\sigma}}=\left\{\sigma_{i j}:\left(\sigma_{i j}-\sigma_{i j}^{0}\right)^{2} \leq\left(\Delta_{i j}^{(E)}\right)^{2}, \quad i=1,2, \ldots, n, j=1,2, \ldots, n\right\} .
$$

We can write the problem (3.12) in bilevel form as:

$$
\text { Upper Level: } \min _{x_{i}}\left\{\frac{1}{2} \sum_{i, j} \sigma_{i j} x_{i} x_{j}: \sum_{i} \mu_{i} x_{i} \geq \tau, \sum_{i} x_{i}=1, x_{i} \geq 0\right\}
$$

$$
\text { Lower Level: } \quad \sigma_{i j} \in \underset{\sigma_{i j}}{\operatorname{argmax}}\left\{\sum_{i, j} \sigma_{i j} x_{i} x_{j}: \sum_{i, j} \frac{\left(\sigma_{i j}-\sigma_{i j}^{0}\right)^{2}}{\left(\Delta_{i j}^{(E)}\right)^{2}} \leq 1\right\}
$$

Again converting the lower level problem into a minimization problem and replacing it by its KKT conditions we have,

$$
\begin{array}{ll}
\min _{x_{i}} \quad & \frac{1}{2} \sum_{i, j} \sigma_{i j} x_{i} x_{j} \\
\text { s.t. } \quad \sum_{i} \mu_{i} x_{i} \geq \tau, \quad \sum_{i} x_{i}=1, \quad x_{i} \geq 0 & \\
\frac{\partial}{\partial \sigma_{i j}} L\left(x_{i}, x_{j}, \sigma_{i j}, \rho\right)=0 \quad \text { where } L\left(x_{i}, x_{j}, \sigma_{i j}, \rho\right)=-\sum_{i, j} \sigma_{i j} x_{i} x_{j}+\rho\left[\sum_{i, j} \frac{\left(\sigma_{i j}-\sigma_{i j}^{0}\right)^{2}}{\left(\Delta_{i j}^{(E)}\right)^{2}}-1\right] \\
\sum_{i, j} \frac{\left(\sigma_{i j}-\sigma_{i j}^{0}\right)^{2}}{\left(\Delta_{i j}^{(E)}\right)^{2}} \leq 1, \quad \rho\left[\sum_{i, j} \frac{\left(\sigma_{i j}-\sigma_{i j}^{0}\right)^{2}}{\left(\Delta_{i j}^{(E)}\right)^{2}}-1\right]=0, \quad \rho \geq 0 \quad \text { for } i, j=1,2, \ldots, n .
\end{array}
$$

Now from the Lagrangian function $L$, we get

$$
\begin{gathered}
\frac{\partial L}{\partial \sigma_{i j}}=-x_{i} x_{j}+2 \rho \frac{\left(\sigma_{i j}-\sigma_{i j}^{0}\right)}{\left(\Delta_{i j}^{(E)}\right)^{2}}=0 \\
\Longrightarrow \sigma_{i j}-\sigma_{i j}^{0}=\frac{x_{i} x_{j}\left(\Delta_{i j}^{(E)}\right)^{2}}{2 \rho}
\end{gathered}
$$

So the single level form (3.19) can be given as:

$$
\begin{array}{ll}
\min _{x_{i}} & \frac{1}{2} \sum_{i, j} \sigma_{i j} x_{i} x_{j} \\
\text { s.t. } \quad & \sum_{i} \mu_{i} x_{i} \geq \tau, \quad \sum_{i} x_{i}=1, \quad x_{i} \geq 0 \\
\sigma_{i j}-\sigma_{i j}^{0}=\frac{x_{i} x_{j}\left(\Delta_{i j}^{(E)}\right)^{2}}{2 \rho}
\end{array}
$$




$$
\begin{aligned}
& \sum_{i, j} \frac{\left(\sigma_{i j}-\sigma_{i j}^{0}\right)^{2}}{\left(\Delta_{i j}^{(E)}\right)^{2}} \leq 1 \\
& \rho\left[\sum_{i, j} \frac{\left(\sigma_{i j}-\sigma_{i j}^{0}\right)^{2}}{\left(\Delta_{i j}^{(E)}\right)^{2}}-1\right]=0 \\
& \rho \geq 0 .
\end{aligned}
$$

Here $\rho$ cannot be zero; otherwise from equation (3.20c) we will get, $\sigma_{i j}-\sigma_{i j}^{0} \rightarrow-\infty$.

So from equation $(3.20 \mathrm{e})$ we get, $\sum_{i, j} \frac{\left(\sigma_{i j}-\sigma_{i j}^{0}\right)^{2}}{\left(\Delta_{i j}^{(E)}\right)^{2}}=1$.

Using equation (3.20c) we get, $\frac{1}{4 \rho^{2}}\left[\sum_{i, j}\left(\Delta_{i j}^{(E)} x_{i} x_{j}\right)^{2}\right]=1 \Longrightarrow \rho=\frac{\sqrt{\sum_{i, j}\left(\Delta_{i j}^{(E)} x_{i} x_{j}\right)^{2}}}{2}$.

Then from (3.20c) we get, $\sigma_{i j}=\sigma_{i j}^{0}+\frac{x_{i} x_{j}\left(\Delta_{i j}^{(E)}\right)^{2}}{\sqrt{\sum_{i, j}\left(\Delta_{i j}^{(E)} x_{i} x_{j}\right)^{2}}}$.

Hence our bilevel problem finally reduces to:

$$
\sqrt{\sum_{i, j}\left(\Delta_{i j}^{(E)} x_{i} x_{j}\right)^{2}}
$$

$$
\begin{array}{ll}
\min _{x_{i}} & \frac{1}{2}\left[\sum_{i, j} \sigma_{i j}^{0} x_{i} x_{j}+\sqrt{\sum_{i, j}\left(\Delta_{i j}^{(E)} x_{i} x_{j}\right)^{2}}\right] \\
\text { s.t. } & \sum_{i} \mu_{i} x_{i} \geq \tau, \quad \sum_{i} x_{i}=1, \quad x_{i} \geq 0 .
\end{array}
$$

\section{Illustrative exAmple}

\subsection{Data description}

For our problem we have considered the monthly return data of four stocks (i) Bajaj Finance, (ii) Reliance Industries Ltd., (iii) Britannia, (iv) Amazon.com Inc. The data are collected from the website https://finance. yahoo. com for the time interval November 2014 to November 2019.

\subsection{Portfolio problem}

We calculate the input parameters like expected return and covariance of return for each asset. Since those values only represent the last 5 years data, so the values for the actual return distribution may vary from our calculated value. That is why we consider the calculated values as the nominal values and we assume some perturbations associated with all those nominal values.

Now the nominal values of the input parameters are calculated as given in Table 1 .

\subsubsection{When uncertainty occurs in expected return terms}

Let the perturbation vector corresponding to the expected return vector is given by,

$$
\boldsymbol{\delta}_{\boldsymbol{\mu}}=\left[\begin{array}{l}
0.03 \% \\
0.04 \% \\
0.05 \% \\
0.06 \%
\end{array}\right]
$$


TABLE 1. Nominal values of input parameters.

\begin{tabular}{lccccc}
\hline \hline \multirow{2}{*}{ Assets } & \multirow{2}{*}{ Expected returns } & \multicolumn{4}{c}{ Covariance of returns } \\
\cline { 3 - 6 } & & Bajaj & Reliance & Britannia & Amazon \\
\hline Bajaj & $4.57 \%$ & $8.622 \%$ & $1.842 \%$ & $2.075 \%$ & $0.039 \%$ \\
Reliance & $3.97 \%$ & $1.842 \%$ & $4.150 \%$ & $1.442 \%$ & $-0.071 \%$ \\
Britannia & $3.11 \%$ & $2.075 \%$ & $1.442 \%$ & $2.092 \%$ & $0.034 \%$ \\
Amazon & $4.22 \%$ & $0.039 \%$ & $-0.071 \%$ & $0.034 \%$ & $0.890 \%$ \\
\hline
\end{tabular}

Then the robust counterpart of our problem is given by,

$$
\begin{array}{ll}
\min _{x_{i}} & \sigma_{P}^{2}=\frac{1}{2}\left[8.622 x_{1}^{2}+4.150 x_{2}^{2}+2.092 x_{3}^{2}+0.890 x_{4}^{2}+2 \cdot 1.842 x_{1} x_{2}+2 \cdot 2.075 x_{1} x_{3}\right. \\
& \left.+2 \cdot 0.039 x_{1} x_{4}+2 \cdot 1.442 x_{2} x_{3}+2 \cdot(-0.071) x_{2} x_{4}+2 \cdot 0.034 x_{3} x_{4}\right] \\
\text { s.t. } & \left\{\min _{\mu \in \mathscr{U}_{\mu}} \sum_{i=1}^{4} \mu_{i} x_{i}\right\} \geq \tau, \quad \sum_{i=1}^{4} x_{i}=1, \quad x_{1}, x_{2}, x_{3}, x_{4} \geq 0 .
\end{array}
$$

(i) Box uncertainty: The robust counterpart problem (4.1) for box uncertainty can be given in bilevel form as:

Upper Level: $\min _{x_{i}}\left\{\sigma_{P}^{2}: \sum_{i=1}^{4} \mu_{i} x_{i} \geq \tau, \quad \sum_{i=1}^{4} x_{i}=1, \quad x_{1}, x_{2}, x_{3}, x_{4} \geq 0\right\}$

s.t.

Lower Level: $\quad \mu_{i} \in \underset{\mu_{i}}{\operatorname{argmin}}\left\{\sum_{i=1}^{4} \mu_{i} x_{i}:\left|\mu_{1}-4.57\right| \leq 0.03,\left|\mu_{2}-3.97\right| \leq 0.04,\left|\mu_{3}-3.11\right| \leq 0.05,\left|\mu_{4}-4.22\right| \leq 0.06\right\}$.

Reducing the above bilevel problem into a single level problem we get the robust counterpart problem as:

$$
\begin{array}{ll}
\min _{x_{i}} \quad \sigma_{P}^{2}=\frac{1}{2}\left[8.622 x_{1}^{2}+4.150 x_{2}^{2}+2.092 x_{3}^{2}+0.890 x_{4}^{2}+2 \cdot 1.842 x_{1} x_{2}+2 \cdot 2.075 x_{1} x_{3}\right. \\
& \left.+2 \cdot 0.039 x_{1} x_{4}+2 \cdot 1.442 x_{2} x_{3}+2 \cdot(-0.071) x_{2} x_{4}+2 \cdot 0.034 x_{3} x_{4}\right] \\
\text { s.t. } & 4.54 x_{1}+3.93 x_{2}+3.06 x_{3}+4.16 x_{4} \geq \tau, \quad \sum_{i=1}^{4} x_{i}=1, \quad x_{1}, x_{2}, x_{3}, x_{4} \geq 0 .
\end{array}
$$

(ii) Ellipsoidal uncertainty: The robust counterpart problem (4.1) for ellipsoidal uncertainty can be given in bilevel form as:

$$
\text { Upper Level: } \min _{x_{i}}\left\{\sigma_{P}^{2}: \sum_{i=1}^{4} \mu_{i} x_{i} \geq \tau, \quad \sum_{i=1}^{4} x_{i}=1, \quad x_{1}, x_{2}, x_{3}, x_{4} \geq 0\right\}
$$

s.t.

Lower Level: $\quad \mu_{i} \in \underset{\mu_{i}}{\operatorname{argmin}}\left\{\sum_{i=1}^{4} \mu_{i} x_{i}: \frac{\left(\mu_{1}-4.57\right)^{2}}{(0.03)^{2}}+\frac{\left(\mu_{2}-3.97\right)^{2}}{(0.04)^{2}}+\frac{\left(\mu_{3}-3.11\right)^{2}}{(0.05)^{2}}+\frac{\left(\mu_{4}-4.22\right)^{2}}{(0.06)^{2}} \leq 1\right\}$.

On reducing the above bilevel problem into a single level problem we get the robust counterpart problem as: 
TABLE 2. Optimal portfolio results for several expected returns when uncertainty affects the expected returns.

\begin{tabular}{|c|c|c|c|c|}
\hline $\begin{array}{l}\text { Expected } \\
\text { return }(\tau)\end{array}$ & Optimal attribute & Nominal data & Box uncertainty & Ellipsoidal uncertainty \\
\hline \multirow{5}{*}{3.8} & $x_{1}$ & 0.000001 & 0.000001 & 0.000012 \\
\hline & $x_{2}$ & 0.081416 & 0.081416 & 0.081416 \\
\hline & $x_{3}$ & 0.227565 & 0.227564 & 0.227552 \\
\hline & $x_{4}$ & 0.691019 & 0.691019 & 0.691020 \\
\hline & Risk & 0.308482 & 0.308482 & 0.308482 \\
\hline \multirow{5}{*}{3.9} & $x_{1}$ & 0.000000 & 0.000000 & 0.000012 \\
\hline & $x_{2}$ & 0.081416 & 0.082486 & 0.081436 \\
\hline & $x_{3}$ & 0.227565 & 0.224661 & 0.227501 \\
\hline & $x_{4}$ & 0.691019 & 0.692853 & 0.691051 \\
\hline & Risk & 0.308482 & 0.308490 & 0.308482 \\
\hline \multirow{5}{*}{4.0} & $x_{1}$ & 0.009983 & 0.024240 & 0.021764 \\
\hline & $x_{2}$ & 0.094371 & 0.106278 & 0.104523 \\
\hline & $x_{3}$ & 0.180091 & 0.131607 & 0.141235 \\
\hline & $x_{4}$ & 0.715555 & 0.737876 & 0.732479 \\
\hline & Risk & 0.310521 & 0.316337 & 0.314889 \\
\hline \multirow{5}{*}{4.1} & $x_{1}$ & 0.034971 & 0.049415 & 0.047926 \\
\hline & $x_{2}$ & 0.115018 & 0.127189 & 0.126380 \\
\hline & $x_{3}$ & 0.093230 & 0.045022 & 0.052633 \\
\hline & $x_{4}$ & 0.756782 & 0.778375 & 0.773060 \\
\hline & Risk & 0.323562 & 0.335938 & 0.333758 \\
\hline \multirow{5}{*}{4.2} & $x_{1}$ & 0.059958 & 0.134762 & 0.128960 \\
\hline & $x_{2}$ & 0.135664 & 0.048738 & 0.066552 \\
\hline & $x_{3}$ & 0.006369 & 0.000000 & 0.000000 \\
\hline & $x_{4}$ & 0.798008 & 0.816500 & 0.804488 \\
\hline & Risk & 0.348488 & 0.393454 & 0.384943 \\
\hline \multirow{5}{*}{4.3} & $x_{1}$ & 0.228571 & 0.368421 & 0.344728 \\
\hline & $x_{2}$ & 0.000000 & 0.000000 & 0.000002 \\
\hline & $x_{3}$ & 0.000000 & 0.000000 & 0.000001 \\
\hline & $x_{4}$ & 0.771429 & 0.631579 & 0.655270 \\
\hline & Risk & 0.496925 & 0.771731 & 0.712192 \\
\hline \multirow{5}{*}{4.4} & $x_{1}$ & 0.514286 & 0.631579 & 0.600001 \\
\hline & $x_{2}$ & 0.000000 & 0.000000 & 0.000000 \\
\hline & $x_{3}$ & 0.000000 & 0.000000 & 0.000001 \\
\hline & $x_{4}$ & 0.485714 & 0.368421 & 0.400000 \\
\hline & Risk & 1.254941 & 1.789100 & 1.632526 \\
\hline
\end{tabular}

$$
\begin{array}{ll}
\min _{x_{i}} & \sigma_{P}^{2}=\frac{1}{2}\left[8.622 x_{1}^{2}+4.150 x_{2}^{2}+2.092 x_{3}^{2}+0.890 x_{4}^{2}+2 \cdot 1.842 x_{1} x_{2}+2 \cdot 2.075 x_{1} x_{3}\right. \\
& \left.+2 \cdot 0.039 x_{1} x_{4}+2 \cdot 1.442 x_{2} x_{3}+2 \cdot(-0.071) x_{2} x_{4}+2 \cdot 0.034 x_{3} x_{4}\right] \\
\text { s.t. } & \left(4.57 x_{1}+3.97 x_{2}+3.11 x_{3}+4.22 x_{4}\right)-\sqrt{(0.03)^{2} x_{1}^{2}+(0.04)^{2} x_{2}^{2}+(0.05)^{2} x_{3}^{2}+(0.06)^{2} x_{4}^{2}} \geq \tau \\
& \sum_{i=1}^{4} x_{i}=1, \quad x_{1}, x_{2}, x_{3}, x_{4} \geq 0 .
\end{array}
$$

Since the portfolio return is somewhere around all the individual asset returns, so we have considered some values in the range from 3.8 to 4.4. For these values of $\tau$, the above two robust counterpart problems are solved 


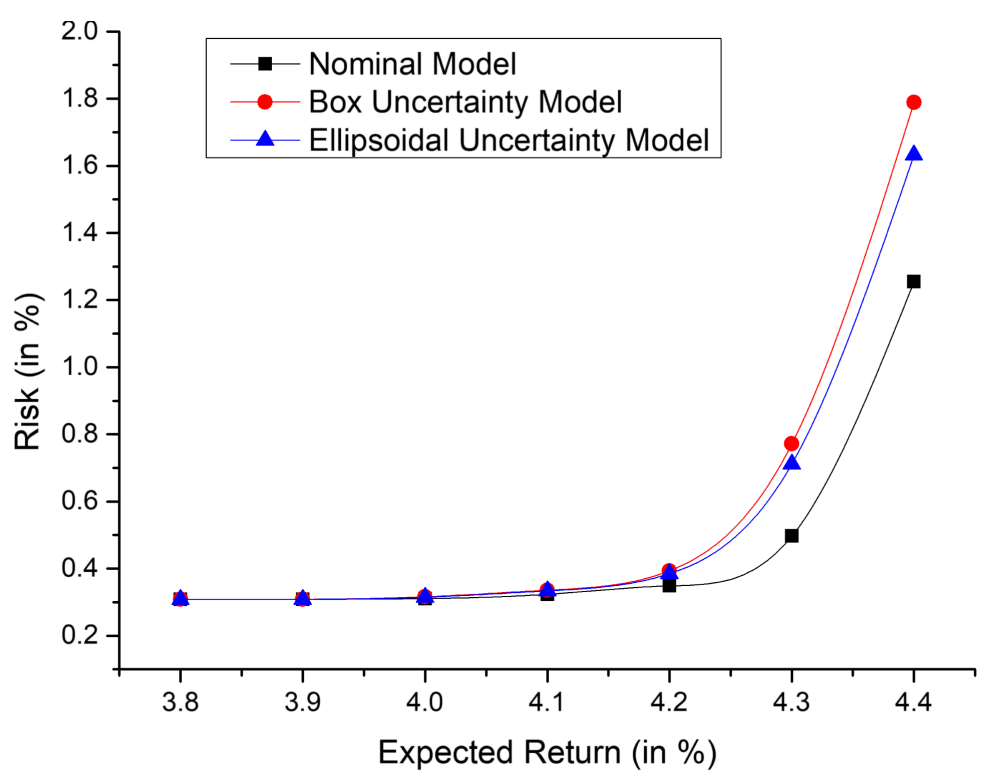

FiguRE 3. Comparison of efficient frontiers for nominal problem and robust problems when uncertainty affects expected returns.

and the results are given in Table 2. From the results, we plot the risk values for different levels of expected returns. This curve is called as the efficient frontier and we plot the efficient frontiers for nominal model as well as box and ellipsoidal uncertainty models, which is shown in Figure 3.

In Figure 3, we can observe that the efficient frontier for the nominal model is at the bottom. This is because in nominal model we ignore uncertainty, so to achieve a certain level of return, less risk is required as compared to the risk required in any uncertain model. Now on comparing efficient frontiers of the two uncertain models, we can observe that the efficient frontier of ellipsoidal model lies below that of box model. That means the investor can achieve a given expected return by taking less risk if the uncertain set is an ellipsoidal one. This confirms that ellipsoidal uncertainty model is less conservative than the box uncertainty model.

\subsubsection{When uncertainty occurs in covariance terms}

Let the perturbation matrix corresponding to the covariance matrix is given by,

$$
\boldsymbol{\delta}_{\boldsymbol{\Sigma}}=\left[\begin{array}{llll}
0.06 \% & 0.00 \% & 0.00 \% & 0.00 \% \\
0.00 \% & 0.05 \% & 0.00 \% & 0.00 \% \\
0.00 \% & 0.00 \% & 0.04 \% & 0.00 \% \\
0.00 \% & 0.00 \% & 0.00 \% & 0.03 \%
\end{array}\right]
$$

Then the robust counterpart of our problem is given by,

$$
\begin{array}{ll}
\min _{x_{i}} \quad\left\{\max _{\Sigma \in \mathscr{U}_{\sigma}} \frac{1}{2} \sum_{i=1}^{4} \sum_{j=1}^{4} \sigma_{i j} x_{i} x_{j}\right\} \\
\text { s.t. } \quad 4.57 x_{1}+3.97 x_{2}+3.11 x_{3}+4.22 x_{4} \geq \tau, \quad \sum_{i=1}^{4} x_{i}=1, \quad x_{1}, x_{2}, x_{3}, x_{4} \geq 0 .
\end{array}
$$


(i) Box uncertainty: The robust counterpart problem (4.4) for box uncertainty can be given in bilevel form as:

Upper Level: $\quad \min _{x_{i}}\left\{\frac{1}{2} \sum_{i=1}^{4} \sum_{j=1}^{4} \sigma_{i j} x_{i} x_{j}: 4.57 x_{1}+3.97 x_{2}+3.11 x_{3}+4.22 x_{4} \geq \tau, \quad \sum_{i=1}^{4} x_{i}=1, \quad x_{1}, x_{2}, x_{3}, x_{4} \geq 0\right\}$ s.t.

Lower Level: $\quad \sigma_{i j} \in \underset{\sigma_{i j}}{\operatorname{argmax}}\left\{\sum_{i=1}^{4} \sum_{j=1}^{4} \sigma_{i j} x_{i} x_{j}:\left|\sigma_{11}-8.622\right| \leq 0.06,\left|\sigma_{22}-4.150\right| \leq 0.05,\left|\sigma_{33}-2.092\right| \leq 0.04\right.$,

$$
\left.\left|\sigma_{44}-0.890\right| \leq 0.03\right\}
$$

TABLE 3. Optimal portfolio results for several expected returns when uncertainty affects the covariance of returns.

\begin{tabular}{|c|c|c|c|c|}
\hline $\begin{array}{l}\text { Expected } \\
\text { return }(\tau)\end{array}$ & Optimal attribute & Nominal data & Box uncertainty & Ellipsoidal uncertainty \\
\hline \multirow{5}{*}{3.8} & $x_{1}$ & 0.000001 & 0.000000 & 0.000013 \\
\hline & $x_{2}$ & 0.081416 & 0.083609 & 0.082905 \\
\hline & $x_{3}$ & 0.227565 & 0.229699 & 0.232814 \\
\hline & $x_{4}$ & 0.691019 & 0.686692 & 0.684268 \\
\hline & Risk & 0.308482 & 0.316815 & 0.315655 \\
\hline \multirow{5}{*}{3.9} & $x_{1}$ & 0.000000 & 0.000000 & 0.000013 \\
\hline & $x_{2}$ & 0.081416 & 0.083609 & 0.082906 \\
\hline & $x_{3}$ & 0.227565 & 0.229699 & 0.232811 \\
\hline & $x_{4}$ & 0.691019 & 0.686692 & 0.684270 \\
\hline & Risk & 0.308482 & 0.316815 & 0.315655 \\
\hline \multirow{5}{*}{4.0} & $x_{1}$ & 0.009983 & 0.011681 & 0.011757 \\
\hline & $x_{2}$ & 0.094371 & 0.096444 & 0.097128 \\
\hline & $x_{3}$ & 0.180091 & 0.180160 & 0.180025 \\
\hline & $x_{4}$ & 0.715555 & 0.711715 & 0.711090 \\
\hline & Risk & 0.310521 & 0.319040 & 0.318185 \\
\hline \multirow{5}{*}{4.1} & $x_{1}$ & 0.034971 & 0.036844 & 0.036880 \\
\hline & $x_{2}$ & 0.115018 & 0.116785 & 0.117874 \\
\hline & $x_{3}$ & 0.093230 & 0.093423 & 0.093187 \\
\hline & $x_{4}$ & 0.756782 & 0.752948 & 0.752059 \\
\hline & Risk & 0.323562 & 0.332658 & 0.332108 \\
\hline \multirow{5}{*}{4.2} & $x_{1}$ & 0.059958 & 0.062007 & 0.061980 \\
\hline & $x_{2}$ & 0.135664 & 0.137126 & 0.138640 \\
\hline & $x_{3}$ & 0.006369 & 0.006686 & 0.006335 \\
\hline & $x_{4}$ & 0.798008 & 0.794181 & 0.793045 \\
\hline & Risk & 0.348488 & 0.358572 & 0.357994 \\
\hline \multirow{5}{*}{4.3} & $x_{1}$ & 0.228571 & 0.228571 & 0.228573 \\
\hline & $x_{2}$ & 0.000000 & 0.000000 & 0.000000 \\
\hline & $x_{3}$ & 0.000000 & 0.000000 & 0.000000 \\
\hline & $x_{4}$ & 0.771429 & 0.771429 & 0.771426 \\
\hline & Risk & 0.496925 & 0.507419 & 0.505989 \\
\hline \multirow{5}{*}{4.4} & $x_{1}$ & 0.514286 & 0.514286 & 0.514287 \\
\hline & $x_{2}$ & 0.000000 & 0.000000 & 0.000000 \\
\hline & $x_{3}$ & 0.000000 & 0.000000 & 0.000000 \\
\hline & $x_{4}$ & 0.485714 & 0.485714 & 0.485712 \\
\hline & Risk & 1.254941 & 1.266415 & 1.263635 \\
\hline
\end{tabular}




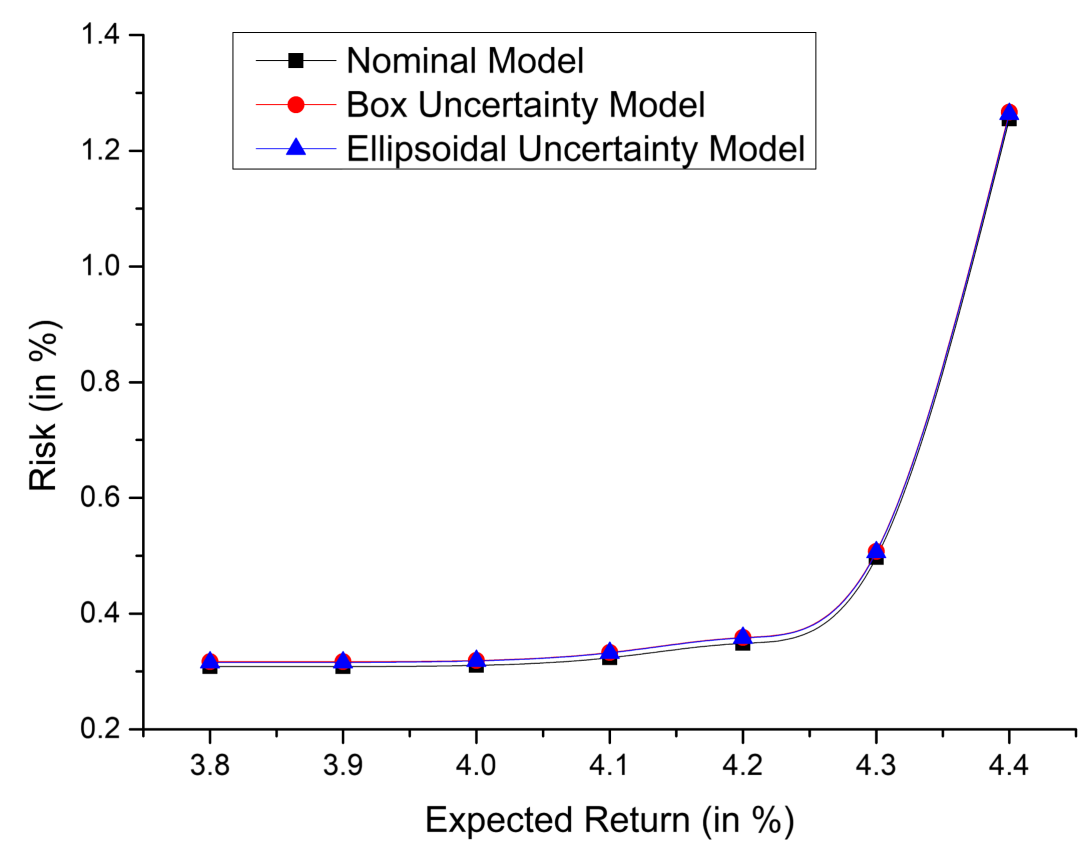

FiguRE 4. Comparison of efficient frontiers for nominal problem and robust problems when uncertainty affects covariance of returns.

On reducing the above bilevel problem into a single level problem we get the robust counterpart problem as:

$$
\begin{array}{ll}
\min _{x_{i}} \quad & \frac{1}{2}\left[8.682 x_{1}^{2}+4.200 x_{2}^{2}+2.132 x_{3}^{2}+0.920 x_{4}^{2}+2 \cdot 1.842 x_{1} x_{2}+2 \cdot 2.075 x_{1} x_{3}\right. \\
& \left.+2 \cdot 0.039 x_{1} x_{4}+2 \cdot 1.442 x_{2} x_{3}+2 \cdot(-0.071) x_{2} x_{4}+2 \cdot 0.034 x_{3} x_{4}\right] \\
\text { s.t. } & 4.57 x_{1}+3.97 x_{2}+3.11 x_{3}+4.22 x_{4} \geq \tau, \quad \sum_{i=1}^{4} x_{i}=1, \quad x_{1}, x_{2}, x_{3}, x_{4} \geq 0 .
\end{array}
$$

(ii) Ellipsoidal uncertainty: The robust counterpart problem (4.4) for ellipsoidal uncertainty can be given in bilevel form as:

Upper Level: $\min _{x_{i}}\left\{\frac{1}{2} \sum_{i=1}^{4} \sum_{j=1}^{4} \sigma_{i j} x_{i} x_{j}: \sum_{i=1}^{4} \mu_{i} x_{i} \geq \tau, \quad \sum_{i=1}^{4} x_{i}=1, \quad x_{1}, x_{2}, x_{3}, x_{4} \geq 0\right\}$

s.t.

Lower Level: $\sigma_{i j} \in \underset{\sigma_{i j}}{\operatorname{argmax}}\left\{\sum_{i=1}^{4} \sum_{j=1}^{4} \sigma_{i j} x_{i} x_{j}: \frac{\left(\sigma_{11}-8.622\right)^{2}}{(0.06)^{2}}+\frac{\left(\sigma_{22}-4.150\right)^{2}}{(0.05)^{2}}+\frac{\left(\sigma_{33}-2.092\right)^{2}}{(0.04)^{2}}+\frac{\left(\sigma_{44}-0.890\right)^{2}}{(0.03)^{2}} \leq 1\right\}$.

On reducing the above bilevel problem into a single level problem we get the robust counterpart problem as:

$$
\begin{array}{ll}
\min _{x_{i}} & \frac{1}{2}\left[8.622 x_{1}^{2}+4.150 x_{2}^{2}+2.092 x_{3}^{2}+0.890 x_{4}^{2}+2 \cdot 1.842 x_{1} x_{2}+2 \cdot 2.075 x_{1} x_{3}+2 \cdot 0.039 x_{1} x_{4}+2 \cdot 1.442 x_{2} x_{3}\right. \\
& \left.+2 \cdot(-0.071) x_{2} x_{4}+2 \cdot 0.034 x_{3} x_{4}+\sqrt{\left(0.06 x_{1}^{2}\right)^{2}+\left(0.05 x_{2}^{2}\right)^{2}+\left(0.04 x_{3}^{2}\right)^{2}+\left(0.03 x_{4}^{2}\right)^{2}}\right] \\
\text { s.t. } & 4.57 x_{1}+3.97 x_{2}+3.11 x_{3}+4.22 x_{4} \geq \tau, \quad \sum_{i=1}^{4} x_{i}=1, \quad x_{1}, x_{2}, x_{3}, x_{4} \geq 0 .
\end{array}
$$


The solutions of the above problems for different values of $\tau$ are given in Table 3 and the efficient frontiers are shown in Figure 4.

From the results of Table 3 it can be observed that the optimal results of the box and ellpsoidal uncertainty models are almost equal to the nominal model. This can also be seen from Figure 4, where efficient frontiers of the uncertain models overlap the nominal model. That means the optimal solution does not get affected very much for the uncertain covariance terms as compared to the uncertain asset returns.

\section{COnClusion}

In this paper, we have proposed a methodology for finding the robust solutions of uncertain portfolio optimization problems. Here we have transformed the uncertain problems into bi-level optimization models and applied the single level reduction approach for deriving their robust counterparts. From the two types of uncertainty sets we have studied, the robust ellipsoidal model is less conservative as compared to the robust box model. In addition, for the uncertain portfolio optimization problems it is better to maintain a tradeoff between optimality and robustness in the solution, so the robust ellipsoidal model can be more useful.

Furthermore, the proposed methodology can be applied for any uncertain minimization problem whose worst case realization of uncertain parameters is a convex problem. There is a scope of further research in the methodology for uncertain multiobjective optimization problems. In future research, we will study the robust counterparts of uncertain portfolio problems under several other uncertainties.

Acknowledgements. The authors would like to thank the editor and the anonymous reviewers for their useful comments and suggestions to improve quality of the paper.

\section{REFERENCES}

[1] M. Asadujjaman and K. Zaman, Robustness-based portfolio optimization under epistemic uncertainty. J. Ind. Eng. Int. 15 (2019) 207-219.

[2] A. Ben-Tal and A. Nemirovski, Robust solutions of uncertain linear programs. Oper. Res. Lett. 25 (1999) 1-13.

[3] A. Ben-Tal and A. Nemirovski, Robust solutions of linear programming problems contaminated with uncertain data. Math. Program. 88 (2000) 411-424.

[4] A. Ben-Tal, L. El Ghaoui, and A. Nemirovski, Robust optimization (Vol. 28). Princeton University Press (2009).

[5] D. Bertsimas, D.B. Brown, and C. Caramanis, Theory and applications of robust optimization. SIAM Rev 53 (2011) 464-501.

[6] Z. Dai and F. Wen, Robust CVaR-based portfolio optimization under a genal affine data perturbation uncertainty set. J. Comput. Anal. Appl. 16 (2014) 93-103.

[7] L. El Ghaoui and H. Lebret, Robust solutions to least-squares problems with uncertain data. SIAM J. Matrix Anal. Appl. 18 (1997) 1035-1064.

[8] L. El Ghaoui, F. Oustry, and H. Lebret, Robust solutions to uncertain semidefinite programs. SIAM J. Optim. 9 (1998) 33-52.

[9] J. Estrada, Systematic risk in emerging markets: the D-CAPM. Emerg. Mark. Rev. 3 (2002) 365-379.

[10] J. Estrada, Mean-semivariance behavior: downside risk and capital asset pricing. Int. Rev. Econ. Finance 16 (2007) $169-185$.

[11] F.J. Fabozzi, D. Huang, and G. Zhou, Robust portfolios: contributions from operations research and finance. Ann. Oper. Res. 176 (2010) 191-220.

[12] J. Fliege and R. Werner, Robust multiobjective optimization \& applications in portfolio optimization. Eur. J. Oper. Res. 234 (2014) 422-433.

[13] D. Goldfarb and G. Iyengar, Robust portfolio selection problems. Math. Oper. Res. 28 (2003) 1-38.

[14] J.H. Kim, W.C. Kim, D.G. Kwon, and F.J. Fabozzi, Robust equity portfolio performance. Ann. Oper. Res. 266 (2018) 293-312 (2018).

[15] Z. Lu, Robust portfolio selection based on a joint ellipsoidal uncertainty set. Optim. Methods EJ Softw. 26 (2011) 89-104.

[16] Z. Lu, K. Deb, and A. Sinha, Uncertainty handling in bilevel optimization for robust and reliable solutions. Int. J. Uncertain. Fuzziness Knowlege-Based Syst. 26 (2018) 1-24.

[17] H. Markowitz, Portfolio selection. J. Finance 7 (1952) 77-91.

[18] H. Markowitz, Portfolio selection: efficient diversification of investments. Basil Blackwell, New York (1959).

[19] S. Nayak and A. Ojha, An approach of fuzzy and TOPSIS to bi-level multi-objective nonlinear fractional programming problem. Soft Comput. 23 (2019) 5605-5618. 
[20] A. Sinha, P. Malo, and K. Deb, A review on bilevel optimization: From classical to evolutionary approaches and applications. IEEE Trans. Evol. Comput. 22 (2017) 276-295.

[21] R.H. Tütüncü and M. Koenig, Robust asset allocation. Ann. Oper. Res. 132 (2004) 157-187.

[22] S. Zhu, D. Li, and S. Wang, Robust portfolio selection under downside risk measures. Quant. Finance 9 (2009) 869-885.

\section{Subscribe to Open (S20) A fair and sustainable open access model}

This journal is currently published in open access under a Subscribe-to-Open model (S2O). S2O is a transformative model that aims to move subscription journals to open access. Open access is the free, immediate, online availability of research articles combined with the rights to use these articles fully in the digital environment. We are thankful to our subscribers and sponsors for making it possible to publish this journal in open access, free of charge for authors.

\section{Please help to maintain this journal in open access!}

Check that your library subscribes to the journal, or make a personal donation to the $\mathrm{S} 2 \mathrm{O}$ programme, by contacting subscribers@edpsciences.org

More information, including a list of sponsors and a financial transparency report, available at: https://www. edpsciences.org/en/maths-s2o-programme 